\title{
Retour vers le futur de l'échange marchand
}

\author{
Myriam Ertz ${ }^{a}$, Damien Hallegatte ${ }^{b}$, Julien Bousquet ${ }^{c}$
}

RÉSUMÉ. Cet article vise à présenter les contributions majeures de l'ouvrage collectif intitulé Les reconfigurations de l'échange marchand: Tour d'borizon, enjeux et perspectives, publié aux Presses universitaires du Québec et dirigé par les professeurs Myriam Ertz, Julien Bousquet et Damien Hallegatte. D’après l'ensemble des collaborateurs de cet ouvrage, l'échange marchand est en mutation profonde. Cette métamorphose a résulté en quatre grandes évolutions: une conceptualisation revue de l'échange marchand, l'émergence de devises d'échanges alternatives, la réactualisation du produit aux cœur d'échange reconfigurés, et la quête de sens dans la consommation. Si les causes de ces évolutions sont assez difficiles à isoler de manière précise, il n'en demeure pas moins que cette modification en profondeur de la manière dont fonctionne le marché résulte de la convergence de trois tendances lourdes : les évolutions technologiques, les évolutions socioculturelles, et les contraintes économico-financières. Cet article résume ainsi les domaines de changement ainsi que les causes de changement tel qu'identifié par les auteurs de l'ouvrage collectif.

\begin{abstract}
This article outlines the major contributions of the collective book entitled Les reconfigurations de l'échange marchand: Tour d'horizon, enjeux et perspectives, published by Presses Universitaires du Québec and edited by professors Myriam Ertr, Julien Bousquet and Damien Hallegatte. According to the contributors of this article, commercial exchange is undergoing a deep change. This transformation led to four main evolutions: a reviewed conceptualization of commercial exchange, the emergence of alternative exchange currencies, updating of the product at the core of re-engineered exchanges, and a quest for a meaning in consumption. If causes of these evolutions are difficult to isolate accurately, it remains that this deep change in the way markets function leads to the convergence of three underlying trends: technological evolutions, sociocultural evolutions, and economic and financial constraints. This article summarizes the areas and the causes of change as identified by the authors of the collective work.
\end{abstract}

\section{Introduction}

Cet article présente les contributions majeures de l'ouvrage collectif intitulé Les reconfigurations de l'échange marchand: Tour d'horizon, enjeux et perspectives, publié en 2019 aux Presses universitaires du Québec. Alors que l'échange marchand a longtemps été confiné à une représentation dichotomique d'une part, avec la distinction nette entre production et consommation, et linéaire, d'autre part, avec l'extraction de ressources, la production, la consommation et la mise au rebut, l'échange marchand est graduellement en train d'être revu non seulement sur le plan de sa représentation conceptuelle, mais également dans sa réalisation concrète. En clair, les manières de se représenter mentalement l'échange évoluent, de même que les manières de réaliser concrètement l'échange.

Ces reconfigurations sont souvent bien documentées dans la littérature par le biais de l'analyse de modèles d'affaires, de systèmes d'échange, d'organisations ou de technologies contribuant à modifier les dynamiques de l'échange marchand (Sundararajan, 2016; Ritzer, 2015; Verhaegen, 2011; Lemaitre et de Barnier, 2015; Sundararajan, 2016; Lambert, 2015; Prahalad et Ramaswamy, 2004; Zwick, Bonsu, et Darmody, 2008; Perren et Kozinets, 2018; Scaraboto, 2015). Toutefois, malgré les apports indéniables de l'ensemble de ces travaux,

\footnotetext{
a Professeure, membre du Labo NFC, Département des sciences économiques et administratives, Université du Québec à Chicoutimi

b Professeur, membre du Labo NFC, Département des sciences économiques et administratives, Université du Québec à Chicoutimi

c Professeur, membre du Labo NFC, Département des sciences économiques et administratives, Université du Québec à Chicoutimi
} 
peu d'études ont synthétisé ces contributions pour en élaborer un cadre analytique plus large comprenant notamment les domaines de reconfigurations de l'échange marchand ainsi que des causes ayant amené à cet état de fait.

L'ouvrage collectif propose de revoir les types de reconfigurations de l'échange marchand ayant eu cours dans un large éventail de contextes et selon des méthodologies variées. Cet article constitue une présentation des contributions et implications principales émanant de l'ouvrage. La première partie fournit un éclaircissement sur la manière dont l'effacement des frontières entre consommation et non-consommation a amené à l'avènement du « tout consommation ». Les auteurs proposent ensuite de faire un retour sur les différents domaines de changements dans l'échange marchand. Enfin, la troisième partie lève le voile sur les causes de la reconfiguration des échanges marchands.

\section{L'effacement des frontières entre consommation et non-consommation : l'avènement imprévu du « tout consommation »}

D'emblée, la préface de l'ouvrage collectif, rédigée par le professeur Bernard Cova, indique que les économies modernes se sont graduellement dirigées vers un "tout consommation» alors même qu'elles tentaient de s'en extraire par divers moyens. Par conséquent, l'économie contemporaine ne s'émanciperait pas de l'omniprésence de la consommation, mais oscillerait entre communautarisation de la consommation et marchandisation de la vie quotidienne.

[Le chercheur en gestion et sciences sociales] s'applique à montrer l'omniprésence de la consommation dans nos vies tout en cherchant à débusquer des voies de sortie du « tout consommation ». Un des phénomènes qui durant les deux dernières décennies a porté à espérer une sortie de ce «tout consommation » a été la montée des collectifs/communautés de consommateurs dans de nombreux secteurs, des loisirs à la santé en passant par l'alimentation. Mais cette montée s'est révélée être à double tranchant, car elle a pu aussi être un parfait «cheval de Troie» de la consommation pour rentrer au plus profond de notre quotidien et s'immiscer dans les moindres replis de notre intimité venant ainsi marchandiser des phénomènes sociaux tels que l'entraide entre individus (Ertz, Bousquet et Hallegatte, 2019, p. VII).

Cette oscillation entre communautarisation de la consommation et marchandisation de la vie quotidienne constitue toutefois le marqueur d'un remodelage plus profond de l'échange marchand. Des communautés de marques aux formes alternatives de consommation fondées sur le partage, la consommation ne se veut plus privée et individualisée, mais s'affiche désormais de manière publique, en groupes, en communautés, et plus largement, en publics de marques. Parallèlement, ce qui était auparavant de l'ordre du privé et de l'intime se marchandise. De nouveaux modèles d'affaires du type Uber, Airbnb, Instacart ou TaskRabbit, fleurons de ce qu'on a appelé « l'économie du partage », visent à marchandiser ce qui était alors de l'ordre de l'entraide et du partage. Par exemple, quel est l'intérêt d'un propriétaire de logement de loger gratuitement un ami ou un étranger via un site d'hébergement temporaire gratuit de personne à personne avec un intermédiaire, comme Couchsurfing par exempt, ou sans intermédiaire, alors qu'il peut monétiser ce service via une plateforme communautaire payante de location et de réservation de logements de particuliers comme Airbnb ou d'autres plateformes du même genre? Il s'agit là de ce que Boltanski et Chiapello (1999) ont appelé la transformation du non-capital en capital. Pour Morozov (2013) il ne s'agit ni plus ni moins que "d'une amplification des pires excès du modèle économique dominant», avec "du néolibéralisme sous stéroïdes ». Quelles que soient les appellations ou les expressions utilisées pour décrire cette reconfiguration des échanges marchands, il n'en demeure pas moins qu'il est difficile de nier ce nouveau phénomène social et économique. Le lecteur se demandera néanmoins ce qui a réellement changé dans l'échange marchand et dans quelle mesure.

\section{Les changement dans l'échange marchand}

En réalité, le monde, en général, et l'économie, en particulier, ne changent pas. Ils se réinventent et se réinterprètent plutôt de manière continue. S’il y a bien un domaine dans lequel ce constat est particulièrement frappant, c'est bien celui de l'échange marchand et de la consommation. Alors que sur le plan conceptuel, il existait une distinction moderniste assez claire entre le monde de la production-distribution d'une 
part, et le monde de l'achat-consommation, d'autre part, dans un monde post-moderne, cette distinction est de moins en moins évidente (Perret, 2015). L'ère postmoderne se caractérise par plus de mouvance, de fluidité, de flexibilité, de liquidité, de rapidité, de manque d'attache ou de référent clair, et où il apparaît de plus en plus commun de ne plus parler uniquement de consommation et de consommateurs, mais de « consommation liquide» (Binkley, 2008), de «prosommation » (Ritzer, 2015), de « consomm'acteurs » (Verhaegen, 2011), de «consomarchands» ou de « consommerçants » (Lemaitre et de Barnier, 2015), de "capitalisme basé sur la foule» (Sundararajan, 2016), de «travail de l'ombre » (Lambert, 2015), de «co-création» (Prahalad et Ramaswamy, 2004), de «travail du consommateur» (Zwick, Bonsu, et Darmody, 2008), de "marché d'échanges latéraux » (Perren et Kozinets, 2018), de «co-production du consommateur» (Dellaert, 2019), ou encore d'« économies hybrides » (Scaraboto, 2015). Il semblerait qu'il y ait désormais un enchâssement plutôt qu'une distinction entre producteur et consommateur.

Le consommateur endosse d'ailleurs une variété de rôles, ce qui le place souvent en amont qu'en aval de la chaine de valeur. L'individu est donc «COproducteur » voire « co-constructeur » (Wood et Ball, 2013), car il endosse des rôles de recycleur, de composteur, de fournisseur (de biens ou de services), ou de réparateurs (Martin et Schouten, 2012). Il est aussi un producteur, car des FabLabs, aux imprimantes 3D, en passant par les jardins communautaires, ou les plateformes de producteurs de type Etsy (site de vente en ligne spécialisé dans les créations personnelles ou le vintage), le consommateur a accès à une palette d'outils qui font (à nouveau) de lui un producteur (Ritzer, 2015). Certains chercheurs ont ainsi revisité le rôle du consommateur en lui reconnaissant une certaine proactivité allant au-delà de son rôle traditionnel consummatif (Ritzer, 2014). Comme le montre bien le chapitre introductif de Patrick Gabriel, c'est ultimement autour de la notion de valeur économique que gravite tout ce phénomène, car l'échange est vise la création de valeur, un terme éminemment complexe, mais qu'il convient de bien définir, afin de saisir toute la portée et l'implication des reconfigurations actuelles de l'échange marchand. En clair, si la création de valeur demeure une réalité fondamentale de l'échange ce sont ses conditions, ses processus, ses systèmes, et ses acteurs qui changent.

Revue Organisations \& Territoires • Volume $28 \cdot \mathrm{N}^{\circ} \mathbf{2} \cdot 2019$
Pour ce qui est de la réinterprétation de l'échange marchand, il s'est donc opéré, conceptuellement, une reconnaissance d'un enchâssement, plutôt que d'une distinction claire, entre les rôles de producteurs et de consommateurs (Perret, 2015; Ritzer, 2015). Cela se vérifie concrètement par une réinvention dans les pratiques. Par exemple, en 2017, il y a eu un record absolu du nombre d'immatriculations de voitures d'occasion en France, alors que l'année 2016 était déjà un record en soi (Autoscout, 2017). L'économie collaborative, plus communément appelée « économie du partage » ou «ubérisation de l'économie », dont la particularité est de permettre aux individus d'endosser le rôle de fournisseur de biens et de services (Belk, 2014), est en progression constante. Alors qu'on ne comptait que 44,8 millions d'utilisateurs de l'économie collaborative, aux États-Unis, en 2016, il y en aura 88,6 millions en 2021 (Statista, 2018). La société de service-conseil PricewaterhouseCoopers a, par ailleurs, estimé que l'économie collaborative devrait passer de 14 milliards de dollars US en 2014 à 335 milliards de dollars US en 2025 (PwC, 2015). Il existerait près de 5000 systèmes de monnaies complémentaires (Magnen et Fourel, 2015), d'origine communautaire, et environ 1855 cryptomonnaies sur des systèmes de pair-à-pair (P2P) (CoinMarketCap, 2018). Les grandes organisations telles que H\&M, Patagonia ou Ikea impliquent également davantage les individus dans des programmes de retour et d'échange de produits usagés (Ertz et al., 2017a, 2017b, 2018). L'enchâssement conceptuel entre le rôle de consommateur et de producteur décrit ainsi une reconfiguration des échanges marchands manifeste dans la praxis (pratique.

Toutefois, s'agit-il réellement d'une nouveauté? L'individu n'a-t-il pas toujours été plus ou moins consommateur et producteur à la fois? C'est du moins ce que se dira intuitivement le public. Cela constituait également le postulat de base de l'école de pensée institutionnelle du marketing (Shaw, 2014; Tadajewski et Jones, 2014), lequel prévalait au début du XXe siècle lorsque le marketing n'en était encore qu'à ses balbutiements. Cette perspective plus macro-orientée et axée sur l'efficacité économique et sociale s'est toutefois perdue dans les années 50 et 60 avec l'apparition d'un paradigme marketing de type managérial, essentiellement axé sur l'entreprise et l'optimisation financière par l'application de méthodes mathématiques et statistiques avancées (Wilkie et Moore, 2003). En Amérique du Nord du moins, la perspective axée sur la firme s'est renforcée avec le temps par le biais des dons émanant d'entreprises et de fondations privées 
accordés aux collèges, écoles de commerces, universités, unités, centres, et chaires de recherche. De multiples avantages ont également été accordés aux chercheurs en marketing afin de résoudre des problématiques managériales. Les noms et les titres des professeurs-chercheurs en marketing, les noms des salles, des bâtiments, et des chaires de recherche, tout ceci démontre de l'influence considérable des entreprises dans l'orientation de la recherche et de la pensée en marketing. S’il est important de demeurer en lien avec le milieu pour ne pas devenir non pertinent, Breyer (1934) rappelle toutefois que le marketing doit avoir une envergure sociétale beaucoup plus large:

Le marketing n'est pas juste un moyen de faire du profit pour les individus. Il s'agit, dans un sens plus large, et plus vital, d'un instrument économique utilisé pour accomplir des objectifs sociaux indispensables... Un système marketing conçu uniquement pour son efficacité sociale transporterait les biens avec un minimum de temps et d'efforts vers les points de déficit. Ce faisant, il fournirait une compensation juste, et sans plus, en contrepartie des efforts de ceux qui sont engagés dans cette activité. De plus, cela fournirait l'incitatif nécessaire pour stimuler des améliorations constantes dans ces méthodes. Il s'agit là des conditions nécessaires pour une efficacité sociale (Beyer, 1934, p. 192, traduction libre).
En accord avec une perspective plus institutionnelle du marketing, en particulier, et de la gestion, en général, l'ouvrage collectif de Ertz, Bousquet et Hallegatte (2019) propose d'effectuer un examen approfondi des nouveaux types d'échange et phénomènes de consommation ayant pour dénominateur commun d'amener à une remise en cause de la distinction traditionnelle entre producteur et consommateur. L'ouvrage démontre comment la consommation se retrouve dans une multiplicité de contextes souvent inédits ou inattendus, tel que le domaine spirituel, par exemple. D'un point de vue normatif, cet examen fournit également des pistes de réflexion sur la question suivante: est-ce que cette plus grande efficacité dans les échanges s'accompagne d'une plus grande efficacité sur le plan social? Les travaux présentés se veulent être des pistes de réflexion pour des recherches futures ainsi que pour des applications pratiques.

Tel que présenté dans la Figure 1, l'ouvrage propose quatre grandes catégories de reconfiguration de l'échange marchand dans lesquelles ont pu se matérialiser les reconfigurations de l'échange marchand, à savoir une conceptualisation revue de l'échange marchand, l'émergence de devises d'échanges alternatives, la réactualisation du produit au cœur d'échanges reconfigurés, et la quête de sens dans la consommation. De plus, ces domaines d'application de la reconfiguration de l'échange marchand résultent de la confluence d'évolutions technologiques, socioculturelles, et économico-financières.

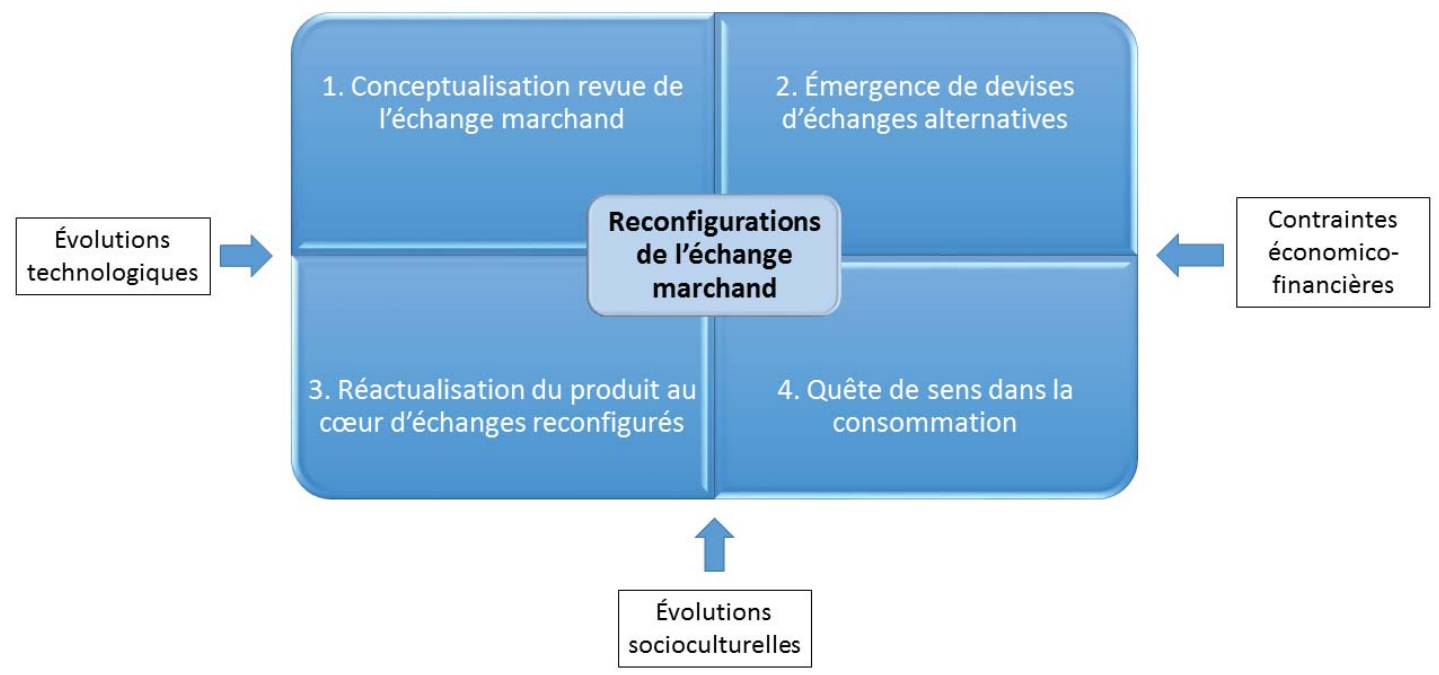

Figure 1-Cadre des reconfigurations de l'échange marchand Source : Ertz, Hallegatte, Bousquet, 2019 


\subsection{Une conceptualisation revue de l'échange marchand}

Les reconfigurations de l'échange marchand ont amené à une nécessité de revisiter les assises conceptuelles ainsi que les perspectives fondamentales à ce sujet de plusieurs auteurs.

\subsubsection{L'échange marchand, une question de création de valeur}

Patrick Gabriel (2019) nous invite à prendre en compte les notions de temps, de sens et de projet pour appréhender un élément fondamental de l'échange marchand: la création de valeur. Après avoir défini la valeur en échange, la valeur expérientielle et la valeur en usage, l'auteur distingue la création de l'évaluation de la valeur. Ces raffinements conceptuels permettent de caractériser l'orientation échange et l'orientation processus de la politique marketing. Dans le premier cas, la valeur en échange ou la valeur expérientielle "participative» se trouvent produites ou coproduites. Dans le deuxième cas, en revanche, ce sont la valeur en usage ou la valeur expérientielle " par appropriation » qui sont créées par une collaboration nécessaire entre une entreprise et un consommateur. Dans un tel parcours de consommation, la valeur n'est pas intrinsèque au produit, mais coconstruite. En outre, notons que la co-création de valeur s'accompagne de co-création de sens, alors que donner un sens à ses activités de consommation fait partie intégrante de l'activité de consommation (Canniford et Shankar, 2013). Gabriel (2019) montre que, par sa politique marketing, l'entreprise propose un système d'échange spécifique, un parcours possible, des ressources pour des projets (identitaires). Ainsi, le type d'échange marchand en soi, par exemple, l'échange dans le marché de l'usagé ou du neuf, en ligne ou hors ligne et ainsi de suite, crée de la valeur, et peut devenir un critère de choix en luimême pour le consommateur. Et reconfigurer l'échange peut être une décision managériale susceptible de créer de la valeur pour chaque partie prenante.

\subsubsection{La reconfiguration des échanges marchands : la prophétie des grands auteurs}

Éric Rémy (2019) met en évidence les apports de trois grands intellectuels français afin de penser la reconfiguration de l'échange marchand en termes d'in- fluences structurelles et institutionnelles, et de déterminismes sociaux. Les écrits du sociologue Émile Durkheim (1858-1917) nous invitent à appréhender la résurgence de la religiosité, à penser la moralisation de la consommation, et à considérer l'évolution des liens sociaux comme un passage «d'une solidarité mécanique à une solidarité organique ». Les travaux du sociologue et psychologue Marcel Mauss (18721950), quant à eux, permettent de penser les limites du don, de la réciprocité, du contrat, de l'utilitarisme, ainsi que de considérer le versant négatif du don, dans une économie numérisée où le consommateur est constamment appelé à travailler, presque toujours bénévolement. Finalement, Rémy (2019) nous propose de considérer la pensée du sociologue Pierre Bourdieu (1930-2002), laquelle permet de mettre en évidence les rapports de forces et de domination, c'est-à-dire de voir l'échange marchand comme un espace de lutte sociale et symbolique. À travers les notions d'habitus, de champ, de goûts, de classes sociales, et de liberté, Bourdieu nous incite à une lecture politique et symbolique de la consommation. Ces trois auteurs rappellent, aux penseurs de la reconfiguration des échanges marchands, le poids des structures et du social, afin d'éviter de grossir les rangs des «idiots utiles » du néolibéralisme, en supposant une liberté accrue des individus (Askegaard, 2014).

\subsection{L'émergence de devises d'échange alternatives}

La reconfiguration des échanges marchands passe aussi par une reconfiguration du panorama des devises d'échange utilisées pour la distribution de biens et de services. Les devises alternatives présentent également l'intérêt de créer des écosystèmes complets d'échanges nouveaux.

\subsubsection{Systèmes d'échanges locaux et monnaies alternatives locales}

Ronan Divard et Patrick Gabriel (2019) se consacrent à l'émergence des monnaies alternatives locales. Les auteurs développent une typologie conceptuelle largement illustrée par de nombreux exemples et rappellent les principaux intérêts liés à cette nouvelle forme d'échange, à savoir soutenir et dynamiser l'économie locale, renforcer les liens sociaux, et faire vivre la citoyenneté. Parallèlement, ils mettent en relief les difficultés de ce phénomène nouveau intimement lié aux consommateurs et à aux valeurs que défendent les consommateurs. Selon les auteurs, pour le 
moment, le développement réel de telles initiatives demeure au stade de potentialité. Les monnaies alternatives s'inscrivent donc dans une tendance plus responsable et locale de la consommation telle que soulevée par d'autres auteurs en marketing (Lecompte et Valette-Florence, 2006), dans des contextes différents. Les auteurs relèvent que les systèmes monétaires simples avec détention préalables tels que les monnaies complémentaires locales ${ }^{1}$ sont plus efficaces que les monnaies-temps ${ }^{2}$ ou les monnaies

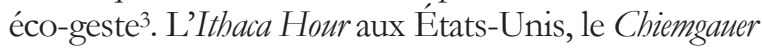
en Allemagne, le Bristol Pound, en Grande - Bretagne ou l'Eusko dans le Pays-Basque, sont les monnaies complémentaires ayant connu le plus grand succès. Toutefois, l'effet actuel des monnaies complémentaires locales demeure très modeste, du moins d'un point de vue quantitatif. Les auteurs concourent avec Blanc (2016) sur le besoin d'une évaluation plus qualitative de leur utilité sociale. Il est intéressant de constater que les monnaies complexes ${ }^{4}$ ne sont pas forcément plus populaires pour autant. La portée intégratrice de leur analyse leur permet ainsi de proposer des pistes pertinentes pour améliorer l'efficacité de ces monnaies alternatives locales.

\subsubsection{Chaîne de blocs et monnaie virtuelle}

Rachid Ghilal et Hamid Nach (2019) débutent leur analyse des monnaies virtuelles par l'importance de la confiance dans le système monétaire. Dans les systèmes financier et monétaire actuels, la confiance est assurée par des intermédiaires faisant, pour l'heure, toujours figure d'autorités centrales reconnues tels que les gouvernements ou les banques, par exemple. Ces intermédiaires permettent de réduire les risques de fraude et d'irrégularité, mais inspirent parfois également de la défiance et en fin de compte un manque de confiance. Toutefois, des développements récents en informatique et en mathématiques ont permis la mise en place d'une technologie appelée chaîne de blocs (blockchain) contournant habilement ces intermédiaires. La chaîne de blocs est à la fois une base de données et un réseau transactionnel de pair-à-pair. De ce fait, elle n'est encadrée par aucune autorité, mais plutôt par l'ensemble des acteurs du réseau. Ce réseau permet la transaction de monnaies dites virtuelles, ou cryptomonnaies, fonctionnant comme de l'argent, mais indépendamment des devises traditionnelles, des frontières nationales et des banques centrales (Maese, Avery, Naftalis, Wink, et Valdez, 2016). Les auteurs précisent également que les cryptomonnaies et leur technologie sous-jacente, la chaine de blocs, sont utilisées dans plusieurs domaines tels que les services financiers, la chaîne d'approvisionnement, ou les services de la santé et gouvernementaux. Les auteurs relèvent les nombreux changements à venir dans les infrastructures digitales ainsi que l'impact de ces dernières sur les contrats, les échanges, et les transactions monétaires. En effet, grâce à la chaîne de blocs, de nombreux domaines tels que la finance, les assurances, ou la fiscalité pourront bénéficier d'une infrastructure transparente et ainsi diminuer le pouvoir des intermédiaires.

\subsubsection{La renaissance du troc}

Kamel Khiari (2019) apporte un regard juridique sur le "paradigme d'échange du troc» (Belk, 2010, p. 715). Tout d'abord, du fait de ses caractéristiques uniques, la notion de troc est complexe en droit civil. En effet, il ne comporte pas le paiement d'un prix. Ensuite, il s'agit d'un échange simultané entre deux biens ou deux services. Dans cette forme authentique, le troc n'est donc pas adapté aux réalités économiques actuelles, car la relation directe d'échange n'est pas toujours possible. Le troc reconfiguré s'appuie sur une nouvelle forme d'échange entre les pairs. Dans ce système, la monnaie intervient à titre d'unité de compte, c'est-à-dire qu'elle est utilisée pour déterminer la valeur des biens. Cette fixation du prix permettra aux parties de fixer la qualité des échanges. Le troc monétisé est une autre sorte de troc reconfiguré, consistant à utiliser la monnaie lorsque deux choses à échanger ne sont pas considérées comme étant égales en termes de valeur. Un troisième groupe de troc configuré se compose de nouvelles monnaies ou des systèmes d'échange alternatifs émergents. Les auteurs soulèvent aussi le recours aux systèmes des points ou d'heure, c'est-à-dire un système d'échange basé sur le troc de biens et de services en utilisant un système de points ou en comptant les heures travaillées. Ces systèmes se rapprochent ainsi des systèmes monétaires simples avec détention préalable dans la typologie de Divard et Gabriel (2019). Enfin, ces motivations à développer de nouveaux modèles d'échange proviennent d'une volonté évidente à améliorer la qualité de vie des membres, à lutter contre la pauvreté, à instaurer un système limitant l'intervention de l'économie de marché. 


\subsection{La réactualisation du produit au cœur d'échanges reconfigurés}

Les biens sont au cœur du système capitaliste moderne. Comme le relevait Karl Marx, dans Das Kapital, le capitalisme a produit un fétichisme de la marchandise. Ce fétichisme provient d'un monde de la production dirigé directement par les producteurs et distributeurs marchands isolés du monde social, et non par la société. C'est de cette parcellisation qu'au $\mathrm{XIX}^{\mathrm{e}}$ siècle, apparut la perspective moderniste de séparation entre production et consommation. Toutefois, le cloisonnement entre la production et la consommation avec la marchandise pour unique vecteur de lien se reconfigure graduellement de plusieurs manières.

\subsubsection{Les bienfaits « insoupçonnés » de la reconfiguration des échanges marchands : l'extension de la durée de vie des produits}

Sébastien Leblanc-Proulx, Myriam Ertz et Emine Sarigöllü (2019) mettent en évidence comment des formes d'échanges alternatives plus variées permettent l'extension de la durée de vie des produits (EDVP). Alors que les relations aux produits ont classiquement été conceptualisées selon la triade «acheter-consommer-jeter», ou plus largement, « extraire-produire-utiliser-jeter ». Ce paradigme «linéaire » a été critiqué, car il induit deux problématiques majeures : 1) du gaspillage et de la pollution (Martin et Schouten, 2012); et 2) une tendance à l'obsolescence programmée, soit un raccourcissement délibéré de la durée de vie des produits afin d'accélérer les taux de remplacement et donc de rachat de ceux-ci (Hellman et Luedicke, 2018). Des voix se sont ainsi élevées pour proposer une "économie circulaire » (Stahel et Reday-Mulvey, 1981), dans laquelle la durée de vie des produits est prolongée grâce à une plus grande diversité des échanges. Si l'atteinte d'une économie réellement circulaire est encore loin de voir le jour, il est inexact d'affirmer que nous évoluons toujours dans une perspective purement linéaire. À partir d'une taxonomie comprenant sept modèles d'affaires permettant d'allonger la durée de vie des produits, Leblanc-Proulx, Ertz, et Sarigöllü (2019) avancent que nous sommes probablement arrivés à un croisement entre perspectives linéaire et circulaire de l'économie. D'une part, cela s'effectue grâce à l'intervention des consommateurs dans les processus organisationnels, notamment par la logistique inverse ou l'utilisation d'un bien comme un service (systèmes de produit-service) plutôt que la possession de ce bien. Cet entrecroisement est également visible dans l'apparition de systèmes souvent d'origines marchandes (ex. eBay) régularisant plus formellement, parfois selon les lois du marché, les pratiques d'échanges des individus, entre eux ou avec les organisations. Au travers de ces processus de co-création de valeur, marqués par l'échange, les consommateurs et les organisations étendent la durée de vie des produits. Ces pratiques permettent ainsi de nous rapprocher d'une économie circulaire. Selon les auteurs, des opportunités de développement d'échange demeurent nombreuses, particulièrement du côté de l'implication des consommateurs.

\subsubsection{Les objets connectés et l'empouvoirement des patients}

Anne-Françoise Audrain-Pontevia, Loick Menvielle et Natascha Adédiran (2019) plongent au cœur des objets connectés (technologies mobiles) et de leurs applications pour les utilisateurs dans le secteur de la santé. Leur recherche vise à comprendre les déterminants de l'intention d'usage des patients quant aux objets connectés, et plus précisément, les effets de trois facteurs contribuant à l'intention d'utiliser les objets connectés dans le domaine de la santé, une pratique qui s'est fortement développée au cours des dernières années. Les chercheurs mettent en évidence la corrélation positive entre la performance attendue, l'effort perçu et l'influence sociale d'un consommateur d'un objet connecté pour la gestion de sa santé avec l'intention d'utiliser cet objet. Les objets connectés, encore mal connus des professionnels de santé apparaissent ainsi comme des outils prometteurs afin d'apporter du confort et un suivi aux patients. Les travaux d'Audrain-Pontevia, Menvielle et Adédiran (2019) s'inscrivent dans l'esprit d'autres recherches (Ouellet, 2016) qui insistent sur le fait que ces technologies nouvelles vont bouleverser nos vies et les façons de faire des organisations, les modèles d'affaires, et donc forcément la consommation.

\subsection{La quête de sens dans la consommation}

Dans un monde postmoderne, la consommation constitue très souvent le projet central de vie d'individus isolés les uns des autres. Toutefois, la consommation est intrinsèquement vide de sens, ce qui amène à un certain désenchantement voire à 
des pathologies de surconsommation aux effets délétères comme... Devant ce vide, certains individus cherchent à recréer du sens et un communautarisme par la consommation, créant de ce fait des communautés de sens.

\subsubsection{Les communautés de familles de patients en ligne : le cas des autistes}

Loick Menvielle, Ghofran Hidri et Anne-Françoise Audrain-Pontevia (2019) s'intéressent à l'impact des communautés virtuelles médicales sur la confiance et l'engagement vis-à-vis du médecin dans les communautés de parents d'enfants atteints de troubles du spectre autistique. La démocratisation des technologies utilisant des outils numériques a permis l'émergence d'espaces dédiés aux patients ou aux proches aidants dans leur recherche d'informations médicales (Akrich et Médal, 2009) déployant de nouvelles formes collaboratives et d'échanges entre individus, donnant naissance au passage à l'émergence d'un nouveau type de patient : «le patient connecté ». Les résultats de cette recherche vont dans le sens des travaux Belanche, Casaló, et Guinalíu (2013), démontrant que la confiance repose sur la qualité des informations délivrées et échangées sur ces plateformes. De plus, la confiance favorise et contribue à l'engagement relationnel vis-à-vis du professionnel de santé et s'avère être nécessaire au maintien d'une bonne qualité des liens entre utilisateurs de ces espaces. Ces résultats s'inscrivent parfaitement dans les tendances associées au marketing participatif (Galibert et Cordelier, 2017) dont l'objectif principal est de maintenir des liens avec utilisateurs, patients ou clients, autrement dit, avec les consommateurs.

\subsubsection{La plance de l'échange dans le domaine religieux}

Dans un monde de croyances en plein bouleversement, les pèlerinages religieux sont en pleine effervescence. Par exemple, celui de Saint-Jacques-deCompostelle renaît dans un engouement général. Véronique Cova, Julien Bousquet et Cylvie Claveau (2019) s'intéressent à la dichotomie sacré/profane de ce pèlerinage en mobilisant une approche historique. Les auteurs montrent qu'à travers les siècles, et plus particulièrement la période du Moyen-Âge, de la Renaissance, et de la Postmodernité, correspondent à une combinaison particulière entre sacré et profane : la séparation entre le sacré et le profane, l'encapsulage des deux et l'hybridation avec le mélange des deux. Cette catégorisation nourrit notre compréhension des pèlerinages en tant que phénomènes sociétaux et marchands. Elle montre que le marché et la consommation ont toujours été présents dans ce pèlerinage mythique, bien qu'ils aient pu évoluer et se modifier au fil du temps. Cette étude fournit un exemple concret du développement et de l'opérationnalisation du concept de sacralisation d'un point de vue marketing (Minton, 2016). Il s'agit d'une thématique ayant donné lieu à tout un courant de recherche dans la discipline depuis près de trois décennies, et plus particulièrement, depuis l'article séminal de Belk, Wallendorf et Sherry (1991).

\subsubsection{Les nouvelles pratiques alimentaires}

Francine Rodier et Caroline Boivin (2019) nous convient à considérer l'évolution des pratiques alimentaires, notamment en termes de consommation d'aliments sains, locaux, en vrac et sousoptimaux (moches, presque périmés). Les motivations des consommateurs peuvent être très variées : d'un côté on retrouve des considérations d'ordre strictement personnel (santé, bien-être, économie parfois...) et de l'autre des considérations très altruistes (condition de travail des producteurs). Répondant à cette demande croissante, la distribution hors épicerie se développe, comme la vente directe du producteur ou du transformateur, les paniers de producteurs, l'autocueillette, ou l'achat autonome en magasin. En développant différentes manières d'entrer en relation avec leurs clients, les producteurs, transformateurs et distributeurs modifient les termes de l'échange marchand. De leur côté, les consommateurs sont à la recherche de pratiques alimentaires ayant un potentiel transformatif élevé, et non récupérables par l'industrie agroalimentaire (Thompson et Coskuner-Balli, 2007). Parmi les évolutions mises en évidence par Rodier et Boivin, c'est peut-être la vente en vrac qui apparaît la plus susceptible de reconfigurer largement et durablement les échanges marchands. Le vrac est une réadaptation moderne de l'image d'Épinal des bons vieux marchés citadins ou provinciaux, dans lesquels le pouvoir des marques est considérablement diminué, alors que l'approvisionnement de la masse des consommateurs est mieux assuré. 


\subsubsection{Phénomènes de consommation et résilience de la société de consommation}

À partir d'un niveau d'analyse plus macro, Damien Hallegatte (2019a) remet en question la portée transformative des consommations collaborative et responsable, afin d'élaborer une critique plus large des potentialités de reconfiguration de l'échange marchand (Dholakia, 2012). Cette analyse a constitué le socle de son essai présentant la société de consommation comme "un piège » (Hallegatte, 2019b). L'auteur met en évidence un ensemble de caractéristiques psychologiques, sociologiques, idéologiques et institutionnelles de la société de consommation, susceptibles de limiter la reconfiguration des échanges marchands, et leur impact réel sur le bien-être des individus. Des changements systémiques importants apparaissent peu probables, en raison notamment du matérialisme, du narcissisme et du cynisme des consommateurs, ainsi que de la logique sociale de la consommation, de l'efficacité du marketing, et de la logique même du marché. Les consommations collaboratives et responsables seraient liberticides, ce qui limiterait leur développement, alors qu'elles n'apporteraient pas le surcroît de pouvoir implicitement promis au consommateur. En outre, l'auteur va jusqu'à soutenir que la consommation responsable est probablement une illusion. En somme, il apparait peu probable que les consommations collaboratives et responsables puissent contribuer de manière importante au progrès humain. Néanmoins, comme tous les chapitres de l'ouvrage, il ouvre au dialogue scientifique sur la reconfiguration des échanges marchands, qui ne fait que commencer.

\section{Les causes}

Dans l'ouvrage, la reconfiguration de l'échange marchand a été appréhendée de manière inédite dans des domaines variés et selon des approches diverses. Cette multidisciplinarité et cette diversité méthodologique a permis de montrer comment la reconfiguration s'opère en termes de réinterprétation conceptuelle et pratique. À la lumière des multiples contributions à cet ouvrage, plusieurs mécanismes et causes sous-jacentes à la reconfiguration de l'échange marchand, peuvent être identifiées. Ces causes sont de nature technologique, sociale et économico-financière.

Revue Organisations $\mathcal{E}$ Territoires $•$ Volume $28 \cdot \mathrm{N}^{\circ}{ }_{2} \cdot 2019$

\subsection{Les évolutions technologiques}

La technologie a joué un rôle capital dans ces changements (Ritzer et Jurgenson, 2010) en permettant aux individus de s'impliquer davantage dans les échanges. Audrain-Pontevia, Menvielle et Adédiran (2019) nous montrent, par exemple, qu'avec l'apparition des objets connectés et des potentialités accrues d'interaction, de monitoring et d'analyse induites par ces objets, les professionnels de santé ont plutôt un rôle de coach et de conseiller, dans une relation moins asymétrique et davantage coconstruite. La technologie a aussi permis de redonner un nouveau souffle à des pratiques généralement considérées comme marginales ou épi-phénoménales à la société de consommation, et appelés systèmes marketing de second ordre (O’Reilly, Rucker, Hughes, Gorang, et Hand, 1984) ou de seconde main (Ertz, Durif, et Arcand, 2017b). Aux brocantes, marchés aux puces, videgreniers, boutiques de seconde main, foires d'église, marchés de troc, enchères, antiquaires, ventes privées du gouvernement, centres de dons, ventes de garage, boîtes de récupération, et aux autres systèmes de logistique inverses de retour des produits et de réutilisation, se sont greffés les plateformes et applications en ligne, attirant des masses critiques d'utilisateurs s'élevant à des millions voire à des milliards d'individus, telles qu'eBay (Chu et Liao, 2007), mais aussi Kijiji, Leboncoin, eBay, la section usagée d'Amazon, VarageSale, Craigslist, Vinted, ou Depop. Au don et au financement ponctuel privé entre particuliers apparaît le prêt de pairs à pairs et le financement participatif (crowdfunding) brassant des milliards de dollars et d'euros; à l'entraide et à l'altruisme traditionnels apparaissent les mouvements de production participative (crowdsourcing); à l'agriculture traditionnelle s'ajoute l'agriculture urbaine, les toits verts, l'agriculture soutenue par la communauté, les Associations pour le Maintien d'une Agriculture Paysanne (AMAPs) et autres mouvements locavores (Martin et Schouten, 2012). Les communautés virtuelles de patients permettent également un rapport nouveau envers les professionnels et le monde médical comme le démontrent Menvielle, Hidri, et Audrian-Pontevia. Enfin, comme le démontre la taxonomie de modèles d'affaires de Leblanc-Proulx, Ertz et Sarigöllü, plusieurs types de modèles d'affaire tels que les systèmes de redistribution par les consommateurs (ex. Craigslist), les courtiers d'accès pair-àpair (ex. Drivy), et les systèmes d'accessibilité gérés 
par des organisations (ex. Bixi), reposent grandement sur le numérique pour permettre l'allongement de la durée de vie des produits. Selon de nombreux travaux, ces remodelages de l'échange marchand ne feront que s'accroître dans les prochaines décennies, à mesure que des avancées supplémentaires dans le domaine des objets connectés, de l'intelligence artificielle, de l'impression 3D ou de la robotique augmenteront encore davantage les capacités des consommateurs (Brynjolfsson et McAfee, 2014; Rust et Huang, 2016; Kumar, Dixit, Javalgi, et Dass, 2014) à s'affranchir des producteurs et des distributeurs ou à co-créer de la valeur avec eux.

\subsection{La quête de sens}

La recherche de sens a constitué un second catalyseur à l'effacement des frontières entre production et consommation. Des communautés de sens émergent afin de redonner de la signification à une société de consommation désenchantée et pour laquelle le réenchantement de la consommation par le point de vente, tel que préconisé par certains auteurs, ne suffit pas (Hawken, 2007). Ce que Paul Hawken a commencé à décrire dès le début des années 2000 ce sont «des congrégations cohérentes, organiques, et organisées, impliquant des dizaines de millions de personnes motivées par le changement» (Hawken, 2007, p.4). Ces congrégations incluent divers groupes, dont le mouvement grandissant de la «santé naturelle » (Thompson et Troester, 2002), dans lequel des personnes avec des objectifs physiques, mentaux, et spirituels communs s'organisent dans des marchés promouvant des «styles de vie de santé et de durabilité » (Lifestyles of health and sustainability [LOHAS]). Les communautés de patients en ligne étudiés par Menvielle, Hidri et Audrain-Pontevia se recoupent avec le mouvement LOHAS sur le plan des préoccupations liées à la santé, mais en diffèrent toutefois par l'usage du nexus des nouvelles technologies, dont les plateformes numériques et les réseaux sociaux. Les circuits courts entre agriculteurs-éleveurs et individus forment une autre manifestation de communauté de sens reliant les producteurs directement aux consommateurs par le biais de systèmes considérés comme étant plus sécuritaires et plus enrichissants que la grande distribution. Rodier et Boivin fournissent un éclaircissement sur ces communautés ainsi que sur les nouvelles configurations d'échange dans ce domaine primordial qu'est l'alimentation. Les communautés de sens structurées s'insèrent souvent dans des mouvements et des pratiques de plus grandes envergures ayant pour dénominateur commun un empouvoirement (empowerment) du consommateur et une critique de la société de surconsommation actuelle. Ces mouvements ont des dénominations et objectifs divers, mais incluent notamment la simplicité volontaire, la permaculture, la décroissance, l'anticonsommation, l'altermondialisme, les plateformes de buycott, comme Carrotmob (Martin et Schouten, 2012), le bashing, et des mouvements de boycott structurés (ex. boycott, désinvestissement, sanction).

Si bon nombre de ces mouvements sont séculaires, ils sont toutefois la manifestation de la recherche d'un certain sens à une consommation, intrinsèquement vide de sens. Dans sa réflexion sur les grands auteurs que sont Durkheim, Mauss et Bourdieu, Rémy explique que, d'un point de vue durkheimien, la simplicité volontaire, le biologique, le commerce équitable, auxquels on peut ajouter la mode lente (slow fashion), la nourriture lente (slow food), la consommation éthique, locale, verte, citoyenne, durable, responsable, et ainsi de suite, sont des manifestations du développement de cadres normatifs dans la consommation, comme des régulations, des carcans sociaux et moraux pour lutter contre l'anomie et contre un individualisme négatif. Pour Rémy, en revisitant Durkheim, le besoin d'expériences et d'émotions collectives profondes où l'on sent que la « société fait corps », traditionnellement assouvi par la religion, l'est désormais de plus en plus par le biais de systèmes de consommation dits « communautaires ». D'où l'émergence et le succès de communautés de sens collaboratives telles que Bookcrossing (Corciolani et Dalli, 2014), Geocaching (Scaraboto, 2015), Couchsurfing (Molz, 2013), le p2p file-sharing (Giesler, 2006), ou même encore le système horizontal de pair-à-pair de la chaîne de blocs et des cryptomonnaies comme le montre le chapitre de Ghilal et Nach.

Parallèlement, alors que la consommation se moralise, le religieux, lui se marchandise par le truchement d'un marketing spirituel ou religieux comme le souligne le chapitre de Cova, Bousquet et Claveau c'est-à-dire qu'il y a "sécularisation du sacré et sacralisation du séculier » (Belk et al., 1991). $\mathrm{Si}$ la conjugaison entre spirituel et marchand a 
toujours constitué un sujet épineux, les auteurs suggèrent que cette distinction s'est relativisée au fil du temps. Historiquement, la combinaison entre sacré et profane est passé de la séparation, à l'hybridation en passant par l'encapsulage. En clair, alors que l'époque faisait clairement le distinguo entre sacré et profane, avec une tolérance toute relative du marchand dans le sacré (séparation), la Renaissance a amené une juxtaposition des deux, avec un accroissement du marchand au détriment du religieux (encapsulage), tandis que l'ère postmoderne, actuelle, se caractérise par une marchandisation généralisée - mise en lumière par les auteurs français de la contreculture depuis les années 1970 (ex. Baudrillard, 1970) - dans laquelle se niche le religieux (hybridation).

\subsection{Les contraintes économico-financière}

Les crises consécutives depuis les années 1970, et en particulier la crise économico-financière de 2008, ont graduellement amené les consommateurs à vivre avec un pouvoir d'achat réduit ou en stagnation. Des salaires atones et une raréfaction du crédit ont donc amené les individus à faire preuve de «braconnage culturel» selon l'expression de Michel de Certeau (cité dans Van de Walle, Hébet et Siounandan, 2012). Les consommateurs ont dû innover pour se procurer les biens et les services dont ils avaient besoin. Ces pratiques ont eu comme particularité d'effacer la dichotomie consommateur - producteur. Toutefois, un examen plus approfondi nous indique également que la cause de cette évolution se trouve dans des chamboulements majeurs observés dans le système économico-financier.

On observe peu à peu une certaine défiance envers des monnaies supranationales telles que l'Euro et la perte de valeur graduelle de certaines devises, notamment du dollar américain face à l'or. Alors qu'au début du XXe siècle, 1000 \$ US permettaient encore d'acheter 52 onces d'or, ceux-ci ne permettent plus que de s'en procurer 0,76 onces en 2011, soit environ 23 grammes (Finance Trends, 2011). Cette chute drastique du pouvoir d'achat exprimé en or n'est que la conséquence d'une succession d'événements marquants, dont la confiscation de l'or des citoyens américains en 1933 par Roosevelt, le Nixon Shock avec la fin de l'étalon-or en 1971 par Richard Nixon (Irwin, 2013), et plus récemment des injections massives de liquidités dans les systèmes financiers par les banques centrales depuis 2008 (Joyce, Lasaosa, Stevens et Tong, 2011). La disparition du référent stable qu'était l'or fit entrer l'Humanité dans l'ère des monnaies fiduciaires, source d'interdépendance monétaire et de spéculation (Frum, 2000), mais aussi d'instabilité financière (Garber, 1993). Ainsi, comme cela fut toujours le cas au cours de l'Histoire, en cas de dépréciation monétaire, comme le système des assignats sous la révolution française (Aubin, 1991), ou l'hyperinflation de la République de Weimar (Laidler et Stadler, 1998), les individus ont progressivement mis en place des systèmes de devises alternatives permettant de se prémunir des risques inhérents à des monnaies en perte de valeur intrinsèque. Selon Divard et Gabriel, ces risques se concentrent en particulier sur la perte de pouvoir d'achat sous l'effet de l'inflation, mais aussi de nombreux autres objectifs comme la destruction de l'économie locale, l'irresponsabilité des organisations, la destruction de lien social ou la perte d'identité. La fin de l'accord de Bretton Woods en 1971, sonnant le glas de l'étalon-or, coïncide d'ailleurs avec l'émergence de tout le courant des devises alternatives, soulignée par Divard et Gabriel :

Les systèmes monétaires alternatifs sont réapparus à partir des années soixante-dix et surtout quatre-vingts sous la forme, non seulement de monnaies locales et complémentaires, mais aussi de systèmes basés sur le temps et qui, dans la grande majorité des cas ne font aucunement référence à la monnaie officielle en circulation (Divard et Gabriel, 2019).

Le chapitre de Ghilal et Nach met en lumière un système sophistiqué de devises alternatives fondées sur la technologie de la chaîne de bloc (blockchain), dénommées les cryptomonnaies. Ces dernières sont apparues dans les années 2010, et ont la particularité d'être gérées de manière décentralisée de pairs à pairs. Elles constituent donc un prolongement technologique aux mouvements d'émancipation et/ou de complémentation des systèmes monétaires conventionnels gérés par les États et les banques centrales. Le chapitre de Khiari prolonge la réflexion sur une pratique en particulier, soit le troc et la manière dont le troc authentique a graduellement laissé la place à un troc moderne reconfiguré dans lequel le recours à la monnaie n'est pas exclu et où des systèmes de monnaies parallèles, de points et d'heures, connexes à l'étude de Divard et Gabriel, ont progressivement fait surface pour faciliter les échanges. La crise financière 
de 2007 a également amené les consommateurs à trouver des alternatives pour se procurer les biens dont ils ont besoin. Ces alternatives comprennent notamment le troc comme le souligne le chapitre de Khiari ou encore l'économie collaborative comprenant le marché de la redistribution et de la mutualisation tel que mis en lumière dans le chapitre de Leblanc-Proulx, Ertz, et Sarigöllü.

\section{Conclusion}

Cet article a présenté les principales contributions de l'ouvrage collectif intitulé Les reconfigurations de l'échange marchand: Tour d'horizon, enjeux et perspectives, publié aux Presses universitaires du Québec et dirigé par les professeurs Myriam Ertz, Julien Bousquet et Damien Hallegatte. En définitive, si les formes de reconfigurations de l'échange marchand sont nombreuses, qu'elles se déclinent dans une variété de domaines, d'organismes, et d'époques, la capacité à réinterpréter et à réimaginer le mieux vivre ensemble propre aux individus demeure une constante et se matérialisera vraisemblablement beaucoup plus fortement encore dans les temps à venir.

\section{NOTES}

1 Les monnaies complémentaires locales sont d'une part complémentaires et locales. Elles sont complémentaires dans le sens où elles ne se substituent pas au système monétaire officiel, mais constituent un complément de ce système, afin de servir des objectifs pour lesquels les monnaies officielles sont trop peu efficaces (ex. soutien de l'économie de proximité). De plus, ces monnaies sont locales car leur aire d'usage est limitée territorialement.

2 Les monnaies-temps consistent en des systèmes de «crédit mutuel » basés sur le temps. L’unité de compte peut être une unité de temps, usuellement l'heure ou une unité spécifique, et cette unité est récompensée en une monnaie spécifique (ex. grain, goutte, piaf, bulle). Par exemple, une heure de travail peut valoir 60 grains.

3 Les monnaies éco-geste visent à promouvoir l'adoption de comportements écoresponsables tels que la réduction de consommation énergétique, le nettoyage de parcs publics, en gratifiant, a posteriori leur auteur d'une récompense monétaire ou autre.

4 Alors que la plupart des systèmes d'échanges alternatifs locaux utilisent un seul étalon (ex. le temps, un geste écoresponsable), les monnaies complexes comportent un plus grand nombre d'étalons (ex. les achats locaux, les pratiques responsables, les achats vertueux

\section{RÉFÉRENCES}

Askegaard, S. (2014). Consumer Culture Theory - Neo-liberalism's 'useful idiots'? Marketing Theory, 14(4), 507-511.

Aubin, C. (1991). Les assignats sous la Révolution française : un exemple d'hyperinflation. Revue Économique, 42(4), 745-761.

Audrain-Pontevia, A.-F., Menvielle, L. et Adédiran, N. (2019). L'étude de trois antécédents de l'intention d'adoption d'objets connectés dédiés à la santé au Québec. Dans M. Ertz, D. Hallegatte, et J. Bousquet (Éds), Les reconfigurations de l'échange marchand: Tour d'horizon, enjeux et perspectives, (pp. 187-205). Québec, QC: Presses de l’Université du Québec.

Autoscout (2017). Baromètre : Marché du véhicule d'occasion en France. Repéré le 31 décembre 2018 à http://about.autoscout24.com/fr-fr/au-press/au-press-news-as24.aspx?pid=588007

Baudrillard, J. (1970). La société de consommation: Ses mythes, ses structures. Paris, France : Gallimard.

Belanche, D., Casaló, L. V. et Guinalíu, M. (2013). The role of consumer happiness in relationship marketing. Journal of Relationship Marketing, 12(2), 79-94.

Belk, R. (2010). Sharing. Journal of Consumer Research, 36, 715-734.

Belk, R. (2014). You are what you can access: Sharing and collaborative consumption online. Journal of Business Research, 67(8), 1595-1600.

Belk, R. W., Wallendorf, M. et Sherry, J. F. (1991). The Sacred and the Profane in Consumer Behavior: Theodicy on the Odyssey. Dans R.Belk (Éd.), Highways and Buyways: Naturalistic Research from the Consumer Behavior Odyssey (pp. 59-101). Provo, UT: Association for Consumer Research.

Binkley, S. (2008). Liquid consumption: Anti-consumerism and the fetishized de-fetishization of commodities. Cultural Studies, 22(5), 599-623. 
Blanc, J. (2016). Les monnaies locales : un bilan d'étape. Paris, France : La Vie des idées.

Breyer, R. F. (1934). The marketing institution. New York, NY: McGraw-Hill.

Brynjolfsson, E. et McAfee, A. (2014). The second machine age: Work, progress, and prosperity in a time of brilliant technologies. New York, NY: WW Norton \& Company.

Canniford, R. et Shankar, A. (2013). Purifying Practices: How Consumers Assemble Romantic Experiences of Nature. Journal of Consumer Research, 39(5), 1051-1069.

Chu, H. et Liao, S. (2007). Exploring consumer resale behavior in C2C online auctions: taxonomy and influences on consumer decisions. Academy of Marketing Science Review, 11(3), 1-27.

CoinMarketCap (2018). Top 100 cryptocurrencies by market capitalization. Repéré le 31 décembre 2018 à https://coinmarketcap.com/

Corciolani, M. et Dalli, D. (2014). Gift-giving, sharing and commodity exchange at Bookcrossing.com: new insights from a qualitative analysis. Management Decision, 52(4), 755-776.

Cova, V., Bousquet, J. et Claveau, C (2019). L'évolution de la dichotomie sacré/profane : une analyse historique du pèlerinage à Saint-Jacques-de-Compostelle. Dans M. Ertz, D. Hallegatte, et J. Bousquet (Éds), Les reconfigurations de l'échange marchand : Tour d'horizon, enjeux et perspectives, (pp. 231-265). Québec, QC : Presses de l’Université du Québec.

Dellaert, B. G. (2019). The consumer production journey: marketing to consumers as co-producers in the sharing economy. Journal of the Academy of Marketing Science, 47(2), 238-254.

Dholakia, N. (2012). Being critical in marketing studies: the imperative of macro perspectives. Journal of Macromarketing, 32(2), $220-225$.

Divard, R. et Gabriel, P. (2019). Les monnaies alternatives locales comme outils d'échanges au service d'objectifs sociétaux. Dans M. Ertz, D. Hallegatte, et J. Bousquet (Éds), Les reconfigurations de l'échange marchand: Tour d'horizon, enjeux et perspectives, (pp. 73-112). Québec, QC : Presses de l’Université du Québec.

Ertz, M., Durif, F. et Arcand, M. (2017a). Life after death? Study of goods multiple lives practices. Journal of Consumer Marketing, 34(2), 108-118.

Ertz, M., Durif, F. et Arcand, M. (2017b). Towards Multilife Marketing: How Goods Multiple Lives Practices Create Value for Consumers. Journal of Promotion Management, 24(6), 863-894.

Ertz, M., Durif, F. et Arcand, M. (2018). Business at the fingertips of consumers: a scale for measuring resale motivations in online settings. The International Review of Retail, Distribution and Consumer Research, 28(1), 92-114.

Ertz, M., Hallegatte, D. et Bousquet, J. (2019). Les reconfigurations de l'échange marchand: Tour d'borizon, enjeux et perspectives. Québec, QC : Presses de l’Université du Québec.

Finance Trends (2011). Purchasing power of US dollar 1920-2009. Repéré le 27 décembre 2018 à https:/ / financetrends.blogspot.com/2011/01/purchasing-power-of-us-dollar-1920-2009.html

Frum, D. (2000). How We Got Here: The 70's. New York, NY: Basic Books.

Gabriel, P. (2019). Les valeurs générées par l'échange marchand : implications dans les orientations des politiques marketing. Dans M. Ertz, D. Hallegatte, et J. Bousquet (Éds), Les reconfigurations de l'échange marchand: Tour d'horizon, enjeux et perspectives, (pp. 17-49). Québec, QC : Presses de l’Université du Québec.

Galibert, O. et B. Cordelier. (2017). Animation et gestion des communautés en ligne : quelles rationalisations du social? Une introduction. Communiquer, 19, 1-8.

Garber, P. M. (1993). The collapse of the Bretton Woods fixed exchange rate system. Dans M. D. Bordo et A. Eichengreen (Éds), A Retrospective on the Bretton Woods System: Lessons for International Monetary Reform (pp. 461-494). Chicago, IL: University of Chicago Press.

Ghilal, R. et Nach, H. (2019). La technologie de la chaine de blocs : fondements et applications. Dans M. Ertz, D. Hallegatte, et J. Bousquet (Éds), Les reconfigurations de l'échange marchand: Tour d'borizon, enjeux et perspectives, (pp. 113-131). Québec, QC : Presses de l’Université du Québec.

Giesler, M. (2006). Consumer gift systems. Journal of Consumer Research. 33(2), 283-290. 
Hallegatte, D. (2019a). Les nouvelles formes de consommation collaborative et responsable : vers une transformation de la société de consummation? Dans M. Ertz, M., D. Hallegatte, et J. Bousquet (Éds), Les reconfigurations de l'échange marchand: Tour d'horizon, enjeux et perspectives, (pp. 281-313). Québec, QC : Presses de l'Université du Québec.

Hallegatte, D. (2019b). Le piège de la société de consommation. Montréal, QC : Liber.

Hawken, P. (2007). Blessed unrest: How the largest movement in the world came into being, and why no one saw it coming, New York, NY: Penguin.

Hellmann, K. U. et Luedicke, M. K. (2018). The Throwaway Society: a Look in the Back Mirror. Journal of Consumer Policy, 41(1), 83-87.

Irwin, D. A. (2013). The Nixon shock after forty years: the import surcharge revisited. World Trade Review, 12(1), 29-56.

Joyce, M., Lasaosa, A., Stevens, I. et Tong, M. (2011). The financial market impact of quantitative easing in the United Kingdom. International Journal of Central Banking, 7(3), 113-161.

Khiari, K. (2019). Le troc authentique et ses erconfigurations face à leurs effets juridiques. Dans M. Ertz, D. Hallegatte, et J. Bousquet (Éds), Les reconfigurations de l'échange marchand: Tour d'horizon, enjeux et perspectives, (pp. 133-152). Québec, QC : Presses de l’Université du Québec.

Kumar, V., Dixit, A., Javalgi, R. R. G. et Dass, M. (2016). Research framework, strategies, and applications of intelligent agent technologies (IATs) in marketing. Journal of the Academy of Marketing Science, 44(1), 24-45.

Laidler, D. E. et Stadler, G. W. (1998). Monetary explanations of the Weimar Republic's hyperinflation: Some neglected contributions in contemporary German literature. Journal of Money, Credit and Banking, 30(4), 816-831.

Lambert, C. (2015). Shadow work: The unpaid, unseen jobs that fill your day. New York, NY: Counterpoint.

Leblanc-Proulx, S., Ertz, M. et Sarigöllü, E. (2019). La taxonomie des modèles d'affaires liés à l'extension de la durée de vie des produits. Dans M. Ertz, D. Hallegatte, et J. Bousquet (Éds), Les reconfigurations de l'échange marchand: Tour d'horizon, enjeux et perspectives, (pp. 155-185). Québec, QC : Presses de l'Université du Québec.

Lecompte, A. F. et P, Valette-Florence (2006). Mieux connaitre le consommateur socialement responsable. Décision Marketing, 41, 67-79.

Lemaitre, N. et De Barnier, V. (2015). Quand le consommateur devient commerçant : motivations, production d'expérience et perspectives. Décisions Marketing, 78, 11-28.

Maese, V. A., Avery, A. W., Naftalis, B. A., Wink, S. P. et Valdez, Y. D. (2016). Cryptocurrency: A primer. The Banking Law Journal, 133, 468-471.

Magnen, J.-P. et C. Fourel (2015). D'autres monnaies pour une nouvelle prospérité. Rapport réalisé à la demande du Ministère du logement, de l'égalité des territoires et de la ruralité et du Secrétariat d'État chargé du commerce, de l'artisanat, de la consommation et de l'économie sociale et solidaire.

Martin, D. et Schouten, J. (2012). Sustainable marketing. Boston, MA: Prentice Hall.

Menvielle, L., Hidri, G. et Audrain-Pontevia, A.-F. (2019). L’impact des communautés virtuelles médicales sur la confiance et l'engagement vis-à-vis du médecin : une application aux communautés de parents d'enfants atteints de troubles du spectre autistique. Dans M. Ertz, D. Hallegatte, et J. Bousquet (Éds), Les reconfigurations de l'échange marchand: Tour d'horizon, enjeux et perspectives, (pp. 209-230). Québec, QC : Presses de l’Université du Québec.

Minton, E. A. (2016). Sacred Attributions: Implications for Marketplace Behavior. Psychology et Marketing, 33(6), 437-448.

Molz, J. G. (2013). Social networking technologies and the moral economy of alternative tourism: The case of couchsurfing.org. Annals of Tourism Research, 43, 210-230.

O’Reilly, L., Rucker, M., Hughes, R., Gorang, M. et Hand, S. (1984). The relationship of psychological and situational variables to usage of a second-order marketing system. Journal of the Academy of Marketing Science, 12(3), 53-76.

Ouellet, J. F. (2016). La révolution de l'intelligence connectée - L'Internet des objets et le marketing en ligne 4.0. Gestion, 41(3), 84-88.

Perren, R. et Kozinets, R. V. (2018). Lateral exchange markets: how social platforms operate in a networked economy. Journal of Marketing, 82(1), 20-36. 
Perret, B. (2015). De la propriété à l’usage. Esprit, 7, 30-39.

Prahalad, C. K. et Ramaswamy, V. (2004). Co-creation experiences: The next practice in value creation. Journal of Interactive Marketing, 18(3), 5-14.

PwC. (2015). The sharing economy. Repéré le 31 décembre 2018 à https://www.pwc.fr/fr/ assets/files/pdf/2015/05/pwc_etude_sharing_economy.pdf

Rémy, É. (2019). Quelles leçons les grands auteurs en sciences humaines et sociales nous donnent-ils sur l'échange marchand contemporain? Réflexions autour de Durkheim, Mauss et Bourdieu. Dans M. Ertz, D. Hallegatte, et J. Bousquet (Éds), Les reconfigurations de l'échange marchand: Tour d'borizon, enjeux et perspectives, (pp. 51-70). Québec, QC : Presses de l'Université du Québec.

Ritzer, G. (2014). Prosumption: Evolution, revolution, or eternal return of the same? Journal of Consumer Culture, 14(1), 3-24.

Ritzer, G. (2015). The "new” world of prosumption: Evolution,"return of the same," or revolution? Sociological Forum, 30(1), $1-17$.

Ritzer, G. et Jurgenson, N. (2010). Production, consumption, prosumption: The nature of capitalism in the age of the digital 'prosumer'. Journal of Consumer Culture, 10(1), 13-36.

Rodier, F. et Boivin, C. (2019). Un tour d'horizon de l'évolution des pratiques alimentaires. Dans M. Ertz, D. Hallegatte, et J. Bousquet (Éds), Les reconfigurations de l'échange marchand: Tour d'horizon, enjeux et perspectives, (pp. 267-279). Québec, QC : Presses de l’Université du Québec.

Rust, R. T. et Huang, M. H. (2014). The service revolution and the transformation of marketing science. Marketing Science, 33(2), 206-221.

Scaraboto, D. (2015). Selling, sharing, and everything in between: The hybrid economies of collaborative networks. Journal of Consumer Research, 42(1), 152-176.

Shaw, E. (2014). The Marketing Institution by Ralph F. Breyer (1934). Journal of Historical Research in Marketing, 6(3), 449-454.

Stahel, W. R. et Reday-Mulvey, G. (1981). Jobs for Tomorrow: The Potential for Substituting Manpower for Energy. New York, NY: Vantage Press.

Statista (2018). Value of the sharing economy worldwide in 2014 and 2025 (in billion USD). Repéré le 31 décembre 2018 à https://www.statista.com/statistics/830986/value-of-the-global-sharing-economy/

Sundararajan, A. (2016). The sharing economy: The end of employment and the rise of crowd-based capitalism. Cambridge, MA: MIT Press.

Tadajewski, M. et Jones, D. B. (2014). Historical research in marketing theory and practice: A review essay. Journal of Marketing Management, 30(11-12), 1239-1291.

Thompson, C. J. et Coskuner-Balli, G. (2007). Countervailing Market Responses to Corporate Co-optation and the Ideological Recruitment of Consumption Communities. Journal of Consumer Research, 34(2), 135-152.

Thompson, C. J. et Troester, M. (2002). Consumer value systems in the age of postmodern fragmentation: The case of the natural health microculture. Journal of Consumer Research, 28(4), 550-571.

Van de Walle, I., Hébel, P. et Siounandan, N. (2012). Les secondes vies des objets : les pratiques d'acquisition et de délaissement des produits de consommation. Cabier de Recherche du Crédoc, nº 290.

Vargo, S. L. et Lusch, R. F. (2004). Evolving to a new dominant logic for marketing. Journal of Marketing, 68(1), 1-17.

Verhagen E. (2011). Le consommateur en tant que consom'acteur. Dans G. Pleyers

(Éd.), La consommation critique : Mouvements pour une alimentation responsable et solidaire, (pp. 259-289). Paris, France : Desclée de Brower.

Wilkie, W. L. et Moore, E. S. (2003). Scholarly research in marketing: Exploring the "4 eras" of thought development. Journal of Public Policy et Marketing, 22(2), 116-146.

Wood, D. M. et Ball, K. (2013). Brandscapes of control? Surveillance, marketing and the co-construction of subjectivity and space in neo-liberal capitalism. Marketing Theory, 13(1), 47-67.

Zwick, D., Bonsu, S. K. et Darmody, A. (2008). Putting Consumers to Work: "Co-Creation" and New Marketing Govern-Mentality. Journal of Consumer Culture, 8(2), 163-196. 